
et dans les oeuvres de ses contemporains

\title{
Rencontre à propos de Peines d'amour perdues
}

Jean-Pierre Vincent et Jean-Michel Déprats

Marie-Thérèse Jones-Davies (éd.)

\section{OpenEdition}

\section{Journals}

Édition électronique

URL : http://journals.openedition.org/shakespeare/134

DOI : $10.4000 /$ shakespeare.134

ISSN : 2271-6424

Éditeur

Société Française Shakespeare

\section{Édition imprimée}

Date de publication : 1 novembre 1980

Pagination : 149-152

\section{Référence électronique}

Jean-Pierre Vincent et Jean-Michel Déprats, "Rencontre à propos de Peines d'amour perdues », Actes des congrès de la Société française Shakespeare [En ligne], 2 | 1980, mis en ligne le 01 novembre 2007, consulté le 02 mai 2019. URL : http://journals.openedition.org/shakespeare/134 ; DOI : 10.4000/ shakespeare.134 


\section{SOCIÉTÉ FRANC̣AISE SHAKESPEARE}

\section{ACTES DU CONGRĖS 1980}

DIRECTEUR DE LA PUBLICATION

M.T. Jones - Davies

JEAN TOUZOT Libraire - Editeur 38 , rue Saint-Sulpice 75278 PARIS CEDEX 061981 


\section{JEUDI 13 NOVEMBRE}

Rencontre avec Jean-Pierre Vincent (metteur en scène), Jean-Michel Déprats (traducteur) à propos de la représentation de Peines d'Amour Perdues donnée au Festival d'Avignon.

La séance commença par l'interprétation par Daniel Briquet, Jean-Louis Fayollet et Emmanuel Schaeffer de deux extraits : Acte III scène $t$ et Acte IV scène irì vers 281 à 384 . Cette illustration scénique fut suivie d'un entretien avec Jean-Pierre Vincent et Jean-Michel Déprats en réponse aux questions de l'auditoire.

J-M.D. : Les traductions existantes s'attachaient surtout à expliciter le sens et les images. Elles conduisaient, ce faisant, soit à allonger la phrase, c'est-à-dire à distendre le ressort poétique et théâtral du texte, soit à banaliser l'étrangeté violente des métaphores paŕ souci de recherche de l'expression française usuelle.

La phrase française a un développement linéaire, elle est fondée sur une architecture grammaticale qui ne totalise le sens qu'à la fin. La phrase de Shakespeare est à l'opposé de cette logique; sa construction est prismatique : l'influx de jeu, l'énergie théâtrale proviennent d'une chaîne presque ininterrompue de mots forts, de pensées et d'images qui forment des constellations et rayonnent alentour. Afin de préserver ces constellations de mots rayonnants, j'ai tenté de serrer de près la construction du texte, sans souci de normalisation sur les codes linguistiques, syntaxiques et lexicaux du français. Plutôt que de chercher à clarifier, j'ai décidé de garder la polysémie, d'assumer la littéralité quand il me semblait important de conserver l'énergie sonore ou mentale d'un mot. Tout en cherchant à garder à peu près le même nombre de mots et le même ordre des mots qu'en anglais, j'ai surtout essayé de maintenir les données formelles, stylistiques et prosodiques du texte. Quitte à sacrifier parfois la précision du lexique à la métrique de l'original, par exemple pour la Chanson du Printemps et de l'Hiver.

J-P.V. : Devant ce type de texte, on peut faire en effet une traduction littéraire destinée à être perçue de façon muette et oculaire par un lecteur. A partir du moment où on veut monter la pièce, il faut se souvenir que c'est un texte écrit 
par un acteur pour des acteurs. Le texte ne résout pas tout. Il doit être complété par un geste, relayé par le corps de l'acteur. Le va-et-vient du mot au geste et du geste au mot ne relève pas d'un rapport mécaniste. Au cours des répétitions, nous avons eu une expérience théâtrale intéressante en travaillant la scène i'de l'Acte III. Au début, les comédiens avaient le sentiment de se trouver devant un rébus indéchiffrable, jusiqu'au jour où ils ont découvert que le texte tissait une série de jeux de mots et de sous-entendus obscènes. Ils se sont mis à tout mimer. Au cours de la septième répétition, la rhétorique gestuelle commençait à se scléroser et l'effet comique à se tarir. Nous avons coupé tous les gestes mais les comédiens étaient tellement nourris de sous-entendus que le texte s'est mis à évoquer tout naturellement une poétique obscène du bas-ventre sans qu'il soit nécessaire de souligner les allusions pour le spectateur.

J-M.D. : Le plaisir de l'invention verbale est une des richesses majeures de cette pièce. Peines d'Amour Perdues est d'une construction dramatique très lâche mais c'est une œuvre d'une prodigalité linguistique étonnante, gorgée de jeux de mots, de polysémie, empourprée de préciosité, fojsonnante d'invention et de création verbale. Curieusement, la critique littéraire s'est peu intéressée à la richesse de cette langue. Elle a surtout cherché à résoudre des énigmes historiques ou à découvrir les intentions satiriques liées à l'identification des personnages. Comme si le seul intérêt de la pièce résidait dans les pastiches qu'elle contient. C'est l'attention portée à la pièce par quelques metteurs en scène de Stratford (notamment Peter Brook) qui a suscité un regain d'intérêt pour une œuvre qui n'est pas, seulement, un exercice de virtuosité formelle.

Jean Jacquot : Je suis sensible à ce que vous dites. Il y a dans cette pièce une extraordinaire qualité de jeunesse, une tension particulière entre la sensibilité et l'esprit.

J-M.D. : Cette jeunesse n'est pas seulement une juvénilité de ton, de comportement et d'atmosphrère.Dans l'utopie active de l'Académie ou dans l'exaltation volontariste du sentiment amoureux, il y a une forme de combat. La jeunesse de l'œuvre, c'est aussi la valeur tonique de cette langue, de ses jeux et de ses enjeux, le questionnement de son emprise sur la réalité, la liberté d'une fonction verbale qui comme chez Joyce ou chez Rabelais construit une vision. 
Gisèle Venet : Il y a une joie transparente de la plasticité des formes, du jeu verbal, un pari sur l'artifice qui est d'une certaine façon le sujet même de la pièce. L'artifice est présent sans que l'on trouve cet arrière-plan, cette mystique en double-fond qui fait l'ambiguïté de La Tempête. La Tempête mise sur l'artifice mais cet artifice est à la fois l'instrument et l'écran de quelque chose qui voudrait se dire et qui finalement ne se dit pas. Dans Peines d'Amour Perdues, l'artifice dit mieux son nom et le dit d'une façon plus totale que dans La Tempête - que finalement je n'aime pas.

J-P.V. : Je suis toujours surpris d'entendre quelqu'un dire qu'il «n'aime pas» une pièce de Shakespeare. Ce ne sont pas des pièces «à aimer», elles sont au-delà de cet état d'âme. Chacune constitue un cosmos indépendant des trente-cinq autres, à la fois refermé sur lui-même et ouvert sur le tout. Comme les pièces d'Eschyle et de Sophocle - et peut-être seulement comme elles - les pièces de Shakespeare se situent dans une autre sphère que toutes les. autres pièces de l'histoire du théâtre.

Raymond Gardette : En quoi sont-elles uniques? Expliquez nous la jubilation théâtrale spécifique d'une pièce de Shakespeare.

J-P.V. : Cette «jubilation» vient d'une relation particulière entre l'acteur et le public, d'un mouvement incessant entre le macrocosme et le microcosme. Les chaines d'images, différentes d'une pièce à l'autre, font traverser à l'acteur et au spectateur des sortes de continents qui se forment, se déforment et évoluent au cours d'un voyage ininterrompu pendant trois heures. Lorsque le texte est dit comme il faut qu'il soit dit, c'est-à-dire à la fois vite et fort, l'acteur se sent plus intelligent et rend le spectateur plus intelligent. J'ai ressenti cette impression pour la première fois chez Peter Brook au cours d'une répétition des deux derniers actes de Timon d'Athènes. Peter Brook avait mis tous les comédiens de dos et côte à côte contre le rideau de fer. Ils devaient dire le texte le plus vite et le plus fort qu'il leur était physiquement possible de le faire. (es deux actes qui à la lecture m'apparaissaient comme une litanie répétitive d'imprécations et de pensées philosophiques devenaient ainsi une sorte de western passionnant. Avec Shakespeare on est au même instant dans l'intériorité d'un être et 
au bout de l'univers, dans la réalité la plus grossière et le raffinement le plus élevé.

Jean-Marie Maguin : Ces chaînes d'images dont vous parlez suggèrent-elles au metteur en scène des images scéniques, des désirs de créer des éclairages, des accessoires, des décors? J-P.V. : A Avignon, nous n'avons pas cherché à utiliser au maximun les ressources scéniques du lieu où nous jouions. I1 y avait simplement le Cloître des Célestins, deux superbes platanes, un plateau recouvert d'une moquette verte. Chez Shakespeare, le décor est dans le texte. Non seulement la localisation de l'action ( Nous sommes dans un parc ou dans un forêt») mais le paysage mental, cette chaîne d'images qui met en branle l'imaginaire du spectateur et permet de passer de l'abstrait au concret. Lorsqu'on joue Shakespeare dans un décor réaliste, il est très difficile de passer à l'abstrait. Inversement, quand on le joue dans un décor abstrait, il est très difficile d'atteindre la réalité des sentiments. Jouer Shakespeare demande un espace libre. La reprise du spectacle en tournée va poser la question du décor de façon aiguë. La scène à l'italienne, inventée en même temps que l'usage de la perspective implique un va-et-vient entre l'image et le mot. Il faut faire un décor. Si, pour sauvegarder la liberté de mouvement, on fait un décor neutre, on n'évite pas l'artifice car la neutralité sur la scène à l'italienne n'est pas neutre. Ce sera peut-être un arbre de dix mètres de haut ou un décor qui raconte le destin de l'Académie. Il faudra faire d'Armado la grande charnière et le centre nerveux de la pièce. 